TRANSACTIONS OF THE

AMERICAN MATHEMATICAL SOCIETY

Volume 352, Number 6, Pages 2857-2869

S 0002-9947(00)02390-4

Article electronically published on February 28, 2000

\title{
ON THE STRUCTURE OF WEIGHT MODULES
}

\author{
IVAN DIMITROV, OLIVIER MATHIEU, AND IVAN PENKOV
}

\begin{abstract}
Given any simple Lie superalgebra $\mathfrak{g}$, we investigate the structure of an arbitrary simple weight $\mathfrak{g}$-module. We introduce two invariants of simple weight modules: the shadow and the small Weyl group. Generalizing results of Fernando and Futorny we show that any simple module is obtained by parabolic induction from a cuspidal module of a Levi subsuperalgebra. Then we classify the cuspidal Levi subsuperalgebras of all simple classical Lie superalgebras and of the Lie superalgebra $\mathbf{W}(n)$. Most of them are simply Levi subalgebras of $\mathfrak{g}_{0}$, in which case the classification of all finite cuspidal representations has recently been carried out by one of us (Mathieu). Our results reduce the classification of the finite simple weight modules over all classical simple Lie superalgebras to classifying the finite cuspidal modules over certain Lie superalgebras which we list explicitly.
\end{abstract}

\section{INTRODUCTION}

In order to be able to explain the topic of this paper and to state the results, we need to start with a few definitions. The remaining definitions needed are given in section 1 . Some of the results apply to arbitrary finite dimensional Lie superalgebras and will be stated in this way.

Consider a finite dimensional Lie superalgebra $\mathfrak{g}=\mathfrak{g}_{0} \oplus \mathfrak{g}_{1}$ defined over an algebraically closed field of characteristic zero. Let $\mathfrak{h}=\mathfrak{h}_{0} \oplus \mathfrak{h}_{1}$ be a Cartan subsuperalgebra and $Q$ be the root lattice. A $\mathfrak{g}$-module $M$ is called a generalized weight module if $M=\bigoplus_{\lambda} M^{(\lambda)}$, where for $\lambda \in \mathfrak{h}_{0}^{*}, M^{(\lambda)}$ denotes the maximal subspace of $M$ on which $h-\lambda(h)$ acts locally nilpotently for any $h \in \mathfrak{h}_{0}$.

By a triangular decomposition $T$ of $\mathfrak{g}$ we mean a decomposition $\mathfrak{g}=\mathfrak{g}_{T}^{+} \oplus \mathfrak{g}_{T}^{0} \oplus \mathfrak{g}_{T}^{-}$ such that there exists a linear map $l: Q \rightarrow \mathbf{Z}$ for which $\mathfrak{g}_{T}^{+}=\bigoplus_{l(\alpha)>0} \mathfrak{g}^{(\alpha)}, \mathfrak{g}_{T}^{0}=$ $\bigoplus_{l(\alpha)=0} \mathfrak{g}^{(\alpha)}$ and $\mathfrak{g}_{T}^{-}=\bigoplus_{l(\alpha)<0} \mathfrak{g}^{(\alpha)}$. This requirement is weaker than the usual one, see for instance [PS]. Correspondingly, we will call the subsuperalgebra $\mathfrak{g}_{T}^{0}$ a Levi subsuperalgebra of $\mathfrak{g}$, or the Levi component of $T$, which will sometimes be in contradiction with the accepted terminology in the theory of Lie algebras.

Any simple generalized weight $\mathfrak{g}_{T}^{0}$-module $\Omega$ can be viewed as a $\mathfrak{g}_{T}^{0} \oplus \mathfrak{g}_{T}^{+}$-module with a trivial action of $\mathfrak{g}_{T}^{+}$. Let $M_{T}(\Omega)=\operatorname{ind}\left(\mathfrak{g}_{T}^{0} \oplus \mathfrak{g}_{T}^{+}, \mathfrak{g} ; \Omega\right)$ be the induced module. It is easy to prove that $M_{T}(\Omega)$ has a unique simple quotient, denoted by $L_{T}(\Omega)$. When the decomposition $T$ is proper (i.e. when $\mathfrak{g}_{T}^{0} \neq \mathfrak{g}$ ), we call the $\mathfrak{g}$ module $L_{T}(\Omega)$ parabolically induced. A cuspidal $\mathfrak{g}$-module is by definition a simple generalized weight module which is not parabolically induced.

Received by the editors October 8, 1997

2000 Mathematics Subject Classification. Primary 17B10.

(C)2000 American Mathematical Society 
Let $M$ be any simple generalized weight module. We associate to $M$ an invariant, called its shadow. This is a triple $\left(\mathfrak{g}_{M}^{+}, \mathfrak{g}_{M}^{I}, \mathfrak{g}_{M}^{-}\right)$consisting of three Lie subsuperalgebras defined in section 3 . We call a triangular decomposition $T$ of $\mathfrak{g} M$-adapted if its Levi component is $\mathfrak{g}_{M}^{I}$ and $\mathfrak{g}_{M}^{ \pm} \subset \mathfrak{g}_{T}^{ \pm}$. The first step of the classification of simple generalized weight modules is the following basic statement (see Theorem 3.6 below):

(T1) For any simple generalized weight $\mathfrak{g}$-module $M$, there is at least one $M$ adapted triangular decomposition $T$ and a cuspidal $\mathfrak{g}_{T}^{0}$-module $\Omega$ such that $M \simeq$ $L_{T}(\Omega)$.

In general, there are many pairs $(T, \Omega)$ such that $M \simeq L_{T}(\Omega)$. To get a more precise statement, one can assume that $\mathfrak{g}_{0}$ is reductive and define certain canonical sets $\mathcal{C}$ of good triangular decompositions (see section 6). For the simple classical Lie superalgebras there are explicit descriptions of such canonical sets. To any simple weight module $M$ we attach then a subgroup $W_{M}$ of the Weyl group $W$ of $\mathfrak{g}_{0}$, called its small Weyl group, see section 5. The following stronger version of (T1) provides an explicit reduction of the classification of simple weight modules to the classification of cuspidal modules.

(T2) For any simple generalized weight $\mathfrak{g}$-module $M$, there is $T \in \mathcal{C}$ and a cuspidal $\mathfrak{g}_{T}^{0}$-module $\Omega$ such that $M \simeq L_{T}(\Omega)$. Moreover the pair $(T, \Omega)$ is unique up to $W_{M}$-conjugacy.

A generalized weight module is called finite if the dimensions of all its weight spaces are finite. The classification of the finite generalized weight modules over all simple Lie algebras is carried out in $[\mathrm{M}]$. We complete the present paper by making the first steps towards classifying all finite generalized weight modules over the simple Lie superalgebras. More precisely, we define a Levi subsuperalgebra to be cuspidal if it admits a finite cuspidal module, and then we classify the cuspidal Levi subsuperalgebras of all classical simple Lie superalgebras. It turns out that most of them are simply Levi subalgebras of $\mathfrak{g}_{0}$, and hence the corresponding cuspidal modules are already classified in $[\mathrm{M}]$. In this way, we reduce the classification problem for the simple finite weight modules over all classical Lie superalgebras to the problem of finding all cuspidal modules over certain Lie superalgebras which are listed in section 8 .

Here is a quick guide through the contents of the paper. In section 1 we fix the notations and section 2 is a brief introduction into the general facts about generalized weight modules. In section 3 we prove (T1). Section 4 contains a cohomological characterization of finite cuspidal modules. From section 5 and on we restrict ourselves to the superalgebras with reductive even part, and in section 5 we introduce the small Weyl group. In section 6 we prove (T2). In the final section 7 we present a complete list of cuspidal Levi subalgebras of all classical simple Lie superalgebras and of $W(n)$.

Remark. When $\mathfrak{g}$ is a reductive Lie algebra and $M$ is finite, the statement (T1) is due to $\mathrm{S}$. Fernando, $[\mathrm{Fe}$. When $\mathfrak{g}$ is a reductive Lie algebra without factors of type $E_{8}$, it is due to V. Futorny, $\mathrm{Fu}$, and A. Cylke, V. Futorny and S. Ovsienko, [CFO]. Our general approach provides a simpler proof.

\section{ACKNOWLEDGMENT}

The support and hospitality of the International Erwin Schrödinger Institute (Vienna) in the Spring of 1996 (where this work was started) isgratefully acknowl- 
edged. We thank G. Benkart, V. Futorny, F. Malikov and V. Serganova for very helpful comments on the subject of this paper. I.D. has been supported partially by NSF Grants DMS 9500755 and INT 9511943, O.M. has been supported by UA1 of the CNRS, and I.P. has been supported partially by NSF Grant DMS 9500755.

\section{Notations and CONVEntions}

1.1. Lie superalgebras. All Lie superalgebras will be defined over an algebraically closed field $K$ of characteristic 0 and will be assumed finite dimensional. All underlying vector spaces of Lie superalgebras and their representations are automatically assumed to be $\mathbf{Z}_{2}$-graded, and the even and odd part of a $\mathbf{Z}_{2}$-graded vector space $V$ will be denoted by $V_{0}$ and $V_{1}$. If $\mathfrak{g}=\mathfrak{g}_{0} \oplus \mathfrak{g}_{1}$ is a Lie superalgebra, a Lie subsuperalgebra $\mathfrak{k} \subset \mathfrak{g}$ will be called a Lie subalgebra iff $\mathfrak{k}_{1}=0$.

1.2. Cartan subsuperalgebras. A Cartan subsuperalgebra $\mathfrak{h}_{0} \oplus \mathfrak{h}_{1}$ of a Lie superalgebra $\mathfrak{g}$ is by definition a self normalizing nilpotent Lie subsuperalgebra of $\mathfrak{g}$. According to Scheunert, [Sch] (see also $\overline{\mathrm{PS}}]$ ), $\mathfrak{h}=\mathfrak{h}_{0} \oplus \mathfrak{h}_{1}$ is a Cartan subsuperalgebra iff $\mathfrak{h}_{0}$ is a Cartan subalgebra of $\mathfrak{g}_{0}$ and $\mathfrak{h}_{1}$ is the generalized weight space of weight 0 of the $\mathfrak{h}_{0}$-module $\mathfrak{g}_{1}$. It should be noted that the definition of a generalized weight module (see the Introduction) involves only the action of $\mathfrak{h}_{0}$.

1.3. Roots $\Delta, \Delta_{0}, \Delta_{1}$. Let $\mathfrak{g}$ be a Lie superalgebra and let $\mathfrak{h}$ be a Cartan subsuperalgebra. For $i \in \mathbf{Z}_{2}$, we denote by $\Delta_{i}$ the set of all $\alpha \in \mathfrak{h}_{0}^{*}$ such that $\alpha \neq 0$ and $\mathfrak{g}_{i}^{(\alpha)} \neq 0$. Set $\Delta:=\Delta_{0} \cup \Delta_{1}$. The elements of $\Delta$ are the roots of $\mathfrak{g}$. A root $\alpha$ is even (respectively odd) if $\alpha \in \Delta_{0}$ (respectively $\alpha \in \Delta_{1}$ ). A root can be simultaneously even and odd. The root lattice $Q$ is the subgroup of $\mathfrak{h}^{*}$ generated by $\Delta$.

1.4. Triangular decompositions. The definition of a triangular decomposition $T$ of $\mathfrak{g}, \mathfrak{g}=\mathfrak{g}_{T}^{+} \oplus \mathfrak{g}_{T}^{0} \oplus \mathfrak{g}_{T}^{-}$, is given in the Introduction. Let $\Delta^{T}=\Delta_{0}^{T} \cup \Delta_{1}^{T}$ be the set of roots of $\mathfrak{g}_{T}^{0}$. We will say that $T$ is a good triangular decomposition iff

(i) the monoid generated by $\Delta_{0}^{T}$ is a group (denoted by $Q_{0}^{T}$ ), and

(ii) for any $\beta \in \Delta_{1}^{T}$, there is $m>0$ so that $m \beta \in Q_{0}^{T}$.

In such a case, we will say that $\mathfrak{g}_{T}^{0}$ is a good Levi subsuperalgebra of $\mathfrak{g}$.

1.5. Dense and torsion-free modules. The support of a generalized weight module $M$ is the set of all $\lambda \in \mathfrak{h}_{0}^{*}$ such that $M^{(\lambda)} \neq 0$, and it is denoted by $\operatorname{supp} M$. We denote by $\operatorname{inj} M$ the set of all roots $\alpha \in \Delta_{0}$ for which there is some $x \in \mathfrak{g}_{0}^{(\alpha)}$ such that $x$ acts injectively on $M$.

We define $M$ to be torsion-free if the monoid generated by $\operatorname{inj} M$ is a subgroup of finite index in $Q$ (Fernando's original definition, [Fe, requires that $\operatorname{inj} M=\Delta$ ). We say that $M$ is dense if $\operatorname{supp} M$ is a finite union of $Q^{\prime}$-cosets, for some subgroup $Q^{\prime}$ of finite index in $Q$ (Futorny's original definition, [Fu], requires that $Q=Q^{\prime}$ ). For simple generalized weight modules, we will show in section 3 that the notions of density and torsion-freeness are both equivalent to cuspidality. With the original definitions of density and torsion-freeness, the latter is true only for finite modules over reductive Lie algebras. 


\section{Generalities about Generalized Weight MOdules}

Let $\mathfrak{g}$ be a Lie superalgebra with a fixed Cartan subsuperalgebra $\mathfrak{h}$ and let $U$ be the enveloping algebra of $\mathfrak{g}$. We denote by $\mathcal{G} W(\mathfrak{g})$ the category of all generalized weight $\mathfrak{g}$-modules and by $\mathcal{G} W_{f}(\mathfrak{g})$ the subcategory of finite generalized weight modules. Throughout the rest of the paper, $M$ will denote a fixed generalized weight $\mathfrak{g}$-module, i.e. an object of $\mathcal{G} W(\mathfrak{g})$. $M$ will not be assumed simple unless the contrary is stated explicitly. We have $U^{(\lambda)} \cdot M^{(\mu)} \subset M^{(\lambda+\mu)}$, for any $\lambda, \mu \in \mathfrak{h}_{0}^{*}$. In particular $U^{(0)}$ is a subalgebra of $U$ and each $U^{(\lambda)}$ is a $U^{(0)}$-bimodule.

Lemma 2.1. (i) Let $\lambda \in Q$. Then $U^{(\lambda)}$ is finitely generated as a left as well as a right $U^{(0)}$-module.

(ii) Let $M \in \mathcal{G} W(\mathfrak{g})$ be simple, let $\lambda \in \operatorname{supp} M$ and let $\beta \in Q$. Then $M^{(\lambda)}$ is a simple $U^{(0)}$-module and $U^{(\beta)} \cdot M^{(\lambda)}=M^{(\lambda+\beta)}$.

Proof. (i) Set $H:=\operatorname{Hom}_{\mathbf{z}}\left(Q, K^{*}\right)$. The torus $H$ acts on $U$ and on its associated graded algebra $S$. The generalized weight space decomposition under $\mathfrak{h}$ is identical with its generalized weight space decomposition under $H$. Hence by Hilbert's invariant theorem, $S^{(0)}$ is finitely generated and each $S^{(\lambda)}$ is a finitely generated $S^{(0)}$-module. This implies that $U^{(\lambda)}$ is finitely generated as a left as well as a right $U^{(0)}$-module.

(ii) Let $m \in M^{(\lambda)}, m \neq 0$. We have $U \cdot m=M$. Hence $U^{(\beta)} \cdot m=M^{(\beta+\lambda)}$, for every $\beta \in Q$. Therefore $U^{(\beta)} \cdot M^{(\lambda)}=M^{(\beta+\lambda)}$, and $M^{(\lambda)}$ is simple as a $U^{(0)}$ module.

A weight module is a module such that $M=\bigoplus_{\lambda} M^{\lambda}$, where $M^{\lambda}$ is the common kernel of $h-\lambda(h)$ for all $h \in \mathfrak{h}_{0}$.

Proposition 2.2. Let $M$ be simple.

(i) $\operatorname{supp} M$ is contained in a single $Q$-coset.

(ii) If $\mathfrak{g}$ is semi-simple as an $\mathfrak{h}_{0}$-module, then $M$ is a weight module.

(iii) If $M^{(\lambda)}$ is finite dimensional for some $\lambda \in \operatorname{supp} M$, then $M$ is finite.

Proof. Assertion (i) follows from Lemma 2.1, (ii). If $\mathfrak{h}_{0}$ acts semi-simply on $\mathfrak{g}$, $\bigoplus_{\lambda} M^{\lambda}$ is obviously a $\mathfrak{g}$-submodule. Thus $M$ is a weight module and Assertion (ii) is proved. Let $\beta \in Q$. By Lemma 2.1,(i) there is a finite set $\left\{u_{1}, \ldots, u_{k}\right\}$ generating the right $U^{(0)}$-module $U^{(\beta)}$. Hence we have

$$
M^{(\beta+\lambda)}=U^{(\beta)} \cdot M^{(\lambda)}=\sum_{1 \leq i \leq k}\left(u_{i} \cdot U^{(0)} \cdot M^{(\lambda)}\right)=\sum_{1 \leq i \leq k}\left(u_{i} \cdot M^{(\lambda)}\right) .
$$

Therefore $\operatorname{dim} M^{(\beta+\lambda)} \leq k \operatorname{dim} M^{(\lambda)}<\infty$, which proves Assertion (iii).

Let $T$ be a triangular decomposition of $\mathfrak{g}$ and let $Q^{T}$ be the root lattice of $\mathfrak{g}_{T}^{0}$. For any object $\Omega$ of $\mathcal{G} W\left(\mathfrak{g}_{T}^{0}\right)$, set $M_{T}(\Omega)=\operatorname{ind}\left(\mathfrak{g}_{T}^{0} \mathfrak{g}_{T}^{+}, \mathfrak{g} ; \Omega\right), \Omega$ being viewed as a $\mathfrak{g}_{T}^{0} \oplus \mathfrak{g}_{T}^{+}$-module with a trivial action of $\mathfrak{g}_{T}^{+}$.

Lemma 2.3. Assume that the support of $\Omega$ is included in a single $Q^{T}$-coset.

(i) The generalized weight $\mathfrak{g}$-module $M_{T}(\Omega)$ is finite iff $\Omega$ is finite.

(ii) There exists a unique maximal $\mathfrak{g}$-submodule $Z_{T}(\Omega)$ in $M_{T}(\Omega)$ such that its intersection with $\Omega$ (considered as a $\mathfrak{g}_{T}^{0} \oplus \mathfrak{g}_{T}^{+}$-submodule in $M_{T}(\Omega)$ ) equals zero.

Proof. Let $l: Q \rightarrow \mathbf{Z}$ be a linear map defining $T$ and let $U^{-}$be the enveloping algebra of $\mathfrak{g}_{T}^{-}$. We have $U^{-}=\bigoplus_{n \geq 0} U^{n}$, where $U^{n}=\bigoplus_{l(\beta)=-n}\left(U^{-}\right)^{\beta}$. Set $M^{\prime}=$ 
$\bigoplus_{n>0} U^{n} \otimes \Omega$. By Poincaré-Birkhoff-Witt's theorem, we have $M_{T}(\Omega) \simeq \Omega \oplus M^{\prime}$ and the supports of $\Omega$ and $M^{\prime}$ are disjoint. Therefore any $\mathfrak{g}$-submodule having trivial intersection with $\Omega$ is a subspace of $M^{\prime}$, and $Z_{T}(\Omega)$ is the sum of all those modules. Assertion (ii) follows. Assertion (i) follows from the fact that each $U^{n}$ is finite dimensional.

For any object $\Omega$ of $\mathcal{G} W\left(\mathfrak{g}_{T}^{0}\right)$ whose support is included in a single $Q^{T}$-coset, we set $L_{T}(\Omega):=M_{T}(\Omega) / Z_{T}(\Omega)$. The correspondence $\Omega \rightsquigarrow L_{T}(\Omega)$ is a kind of HarishChandra induction functor. The corresponding Harish-Chandra restriction is the functor $M \rightsquigarrow H^{0}\left(\mathfrak{g}_{T}^{+}, M\right)$.

Corollary 2.4. Let $\Omega$ be an object of $\mathcal{G} W\left(\mathfrak{g}_{T}^{0}\right)$ whose support is contained in a single $Q^{T}$-coset.

(i) $H^{0}\left(\mathfrak{g}_{T}^{+}, L_{T}(\Omega)\right)=\Omega$.

(ii) $H_{0}\left(\mathfrak{g}_{T}^{-}, L_{T}(\Omega)\right)=\Omega$.

(iii) $\Omega$ is simple iff $L_{T}(\Omega)$ is simple.

(iv) $\Omega$ is finite iff $L_{T}(\Omega)$ is finite.

Proof. Let $v$ be a generalized weight vector in $H^{0}\left(\mathfrak{g}_{T}^{+}, L_{T}(\Omega)\right)$. If $v \notin \Omega$, then the support of the $\mathfrak{g}$-submodule of $L_{T}(\Omega)$ generated by $v$ is disjoint from the support of $\Omega$, which is a contradiction. This proves Assertion (i). The proof of Assertion (ii) is similar. Any non-zero submodule of $L_{T}(\Omega)$ intersects $\Omega$ non-trivially, and $\Omega$ generates $L_{T}(\Omega)$. Hence $L_{T}(\Omega)$ is simple whenever $\Omega$ is simple. For any $\mathfrak{g}_{T^{-}}^{0}$ submodule $\Omega^{\prime} \subset \Omega$, we have $L_{T}\left(\Omega^{\prime}\right) \subset L_{T}(\Omega)$, which proves also that $\Omega$ is simple whenever $L_{T}(\Omega)$ is simple. Assertion (iii) is proved. Assertion (iv) follows from Lemma 2.3,(ii) and from the fact that $\Omega \subset L_{T}(\Omega)$.

\section{The Shadow and a generalization of Fernando-Futorny's theorem}

Lemma 3.1. Let $M$ be simple and let $x \in \mathfrak{g}_{0}^{\alpha}$ for some $\alpha \in \Delta_{0}$. Then $x: M \rightarrow M$ is either injective or locally nilpotent.

Proof. The set $M^{\prime}$ of all vectors $m \in M$ such that $x^{N} \cdot m=0$ for some $N \geq 1$ is a $\mathfrak{g}$-submodule. If $M^{\prime}=0$, then $x$ acts injectively. Otherwise $M^{\prime}=M$ and $x$ acts locally nilpotently.

We define a cone as a finitely generated submonoid of $Q$. For any cone $C$, its saturation $\bar{C}$ is the cone of all $\lambda \in Q$ such that $m \lambda \in C$ for some positive integer $m$. Any cone contains a maximal subgroup $G(C)(G(C)$ is nothing but $C \cap-C)$ and we have $G(\bar{C})=\overline{G(C)}$. For any $M$, we denote by $C_{M}^{1}$ the cone generated by inj $M$, and by $C_{M}^{2}$ the set of all $\beta \in Q$ such that $\beta+\operatorname{supp} M \subset \operatorname{supp} M$. It is obvious that $C_{M}^{1} \subset C_{M}^{2}$.

Proposition 3.2. Let $M$ be simple. There is a finite set $\Theta$ such that $\operatorname{supp} M=$ $C_{M}^{1}+\Theta$. In particular $\overline{C_{M}^{1}}=\overline{C_{M}^{2}}$.

Proof. For any $i \in \mathbf{Z}_{2}$ and any $\alpha \in \Delta_{i}$, choose a basis $B_{i}^{\alpha}=\left\{x_{i, 1}^{\alpha}, x_{i, 2}^{\alpha}, \ldots\right\}$ of $\mathfrak{g}_{i}^{\alpha}$, with the requirement that $x_{i, 1}^{\alpha}$ acts injectively on $M$ whenever $\alpha$ belongs to inj $M$. Let $U(\alpha)$ be the span of all monomials $\left(x_{i, 1}^{\alpha}\right)^{m_{1}} \cdot\left(x_{i, 2}^{\alpha}\right)^{m_{2}} \cdot \ldots \cdot$ where $i \in \mathbf{Z}_{2}$ is such that $\Delta_{i} \neq \emptyset$, and $m_{1}, m_{2}, \ldots$ are non-negative integers not exceeding 1 in case $i=1$.

Set $j=\# \operatorname{inj} M, a=\# \Delta_{0}, b=\# \Delta_{1}$ ( \# standing for cardinality) and write $\Delta_{0} \cup \Delta_{1}=\left\{\alpha_{1}, \alpha_{2}, \ldots, \alpha_{a+b}\right\}$, where the first $j$ roots are the roots in $\operatorname{inj} M$ and 
the first $a$ roots are the roots in $\Delta_{0}$. Choose furthermore $\lambda \in \operatorname{supp} M$, put $N:=$ $U\left(\alpha_{j+1}\right) \cdot U\left(\alpha_{j+2}\right) \cdots \cdot U\left(\alpha_{a+b}\right) \cdot M^{(\lambda)}$ and let $\Theta$ be the support of the finite dimensional $\mathfrak{h}_{0}$-module $N$. By Poincare-Birkhoff-Witt's theorem, we have $M=$ $U\left(\alpha_{1}\right) \cdot U\left(\alpha_{2}\right) \cdot \ldots \cdot U\left(\alpha_{j}\right) \cdot N$, and therefore supp $M=C_{M}^{1}+\Theta$. This implies that the saturations of $C_{M}^{1}$ and $C_{M}^{2}$ are the same.

In what follows we will denote the saturation of the cones $C_{M}^{1}$ and $C_{M}^{2}$ by $C_{M}$ and will call it the cone of $M$. Using the cone $C_{M}$ ( $M$ being assumed simple) one can decompose $\Delta$ into four disjoint sets $\Delta_{M}^{I}, \Delta_{M}^{F}, \Delta_{M}^{+}$and $\Delta_{M}^{-}$, defined as follows:

$$
\begin{aligned}
& \Delta_{M}^{I}:=\left\{\alpha \in \Delta \mid \alpha \in C_{M},-\alpha \in C_{M}\right\}, \\
& \Delta_{M}^{F}:=\left\{\alpha \in \Delta \mid \alpha \notin C_{M},-\alpha \notin C_{M}\right\}, \\
& \Delta_{M}^{+}:=\left\{\alpha \in \Delta \mid \alpha \notin C_{M},-\alpha \in C_{M}\right\}, \\
& \Delta_{M}^{-}:=\left\{\alpha \in \Delta \mid \alpha \in C_{M},-\alpha \notin C_{M}\right\} .
\end{aligned}
$$

The decomposition $\Delta=\Delta_{M}^{I} \sqcup \Delta_{M}^{F} \sqcup \Delta_{M}^{+} \sqcup \Delta_{M}^{-}$is by definition the $M$-decomposition of $\Delta$. Recalling that if $\alpha \in \Delta$ and $\lambda \in \operatorname{supp} M$, the $\alpha$-string through $\lambda$ is the set $\{x \in \mathbf{Q} \mid \lambda+x \alpha \in \operatorname{supp} M\}$, one verifies

Lemma 3.3. (i) $\alpha \in \Delta_{M}^{F}$ iff the $\alpha$-string through any $\lambda \in \operatorname{supp} M$ is bounded;

(ii) $\alpha \in \Delta_{M}^{I}$ iff the $\alpha$-string through any $\lambda \in \operatorname{supp} M$ is unbounded in both directions;

(iii) $\alpha \in \Delta_{M}^{+}$iff the $\alpha$-string through any $\lambda \in \operatorname{supp} M$ is bounded from above only;

(iv) $\alpha \in \Delta_{M}^{-}$iff the $\alpha$-string through any $\lambda \in \operatorname{supp} M$ is bounded from below only.

Corollary 3.4. If $M$ is simple, the $\alpha$-string through $\lambda$ is bounded (respectively bounded from above only, bounded from below only, or unbounded in both directions) for every $\lambda \in \operatorname{supp} M$ whenever it is bounded (respectively bounded from above only, bounded from below only, or unbounded in both directions) for some $\mu \in \operatorname{supp} M$.

It is an immediate consequence from the definition of the $M$-decomposition that the following three subspaces of $\mathfrak{g}$

$$
\mathfrak{g}_{M}^{+}:=\bigoplus_{\alpha \in \Delta_{M}^{+}} \mathfrak{g}^{(\alpha)}, \quad \mathfrak{g}_{M}^{I}:=\mathfrak{h} \oplus\left(\bigoplus_{\alpha \in \Delta_{M}^{I}} \mathfrak{g}^{(\alpha)}\right), \quad \mathfrak{g}_{M}^{-}:=\bigoplus_{\alpha \in \Delta_{M}^{-}} \mathfrak{g}^{(\alpha)}
$$

are Lie subsuperalgebras of $\mathfrak{g}$. We define the shadow of $M$ onto $\mathfrak{g}$ (or simply the shadow of $M)$ as the triple $(*)$. It is clear that the shadow of $M$ reconstructs the $M$-decomposition of $\Delta$ and vice versa.

We are ready now to prove a generalization of Theorem 4.18 in $[\mathrm{Fe}$ as well as of the main result of [CFO]. We start with

Lemma 3.5. Let $C \subset Q$ be a saturated cone and let $D \subset Q$ be a finite subset. There is a linear form $l: Q \rightarrow \mathbf{Z}$ such that $\operatorname{ker} l \cap(C \cup D)=G(C)$.

Proof. Exercise which uses the convexity of $C$.

Recalling (see the Introduction) that a triangular decomposition $T$ of $\mathfrak{g}$ is $M$ adapted if $\mathfrak{g}_{M}^{I}=\mathfrak{g}_{T}^{0}$ and $\mathfrak{g}_{M}^{ \pm} \subset \mathfrak{g}_{T}^{ \pm}$, we have

Theorem 3.6. Let $M$ be a simple generalized weight $\mathfrak{g}$-module.

(i) $\mathfrak{g}_{M}^{I}$ is a good Levi subsuperalgebra of $\mathfrak{g}$.

(ii) There is at least one and at most finitely many $M$-adapted triangular decompositions $T$. 
(iii) For any adapted triangular decomposition $T$, there exists a unique cuspidal $\mathfrak{g}_{M}^{I}$-module $\Omega$ such that $M \simeq L_{T}(\Omega)$.

Proof. Set inv $M=\operatorname{inj} M \cap \Delta_{M}^{I}$. Assertion (i) follows from the fact that the monoid generated by $\operatorname{inv} M$ is the group $G\left(C_{M}^{1}\right)$, which is of finite index in the root lattice $Q^{T}$ of $\mathfrak{g}_{M}^{I}$.

The existence of an $M$-adapted triangular decomposition follows from Lemma 3.3. There are only finitely many triangular decompositions, so only finitely many are $M$-adapted. Assertion (ii) is proved.

It remains to prove Assertion (iii). Let $l: Q \rightarrow \mathbf{Z}$ be a linear map defining the $M$-adapted triangular decomposition $T$ and let $X$ be the $Q$-coset containing $\operatorname{supp} M$. There is a map $d: X \rightarrow \mathbf{Z}$ such that $d(\lambda+\beta)=d(\lambda)+l(\beta)$ for any $\lambda \in X, \beta \in Q$. By the definition of $l$ we have $l(\beta) \leq 0$ for any $\beta \in C_{M}^{1}$. As supp $M=C_{M}^{1}+\Theta$ for some finite set $\Theta$, the function $d$ is bounded from above on $\operatorname{supp} M$. Set $\Omega=\bigoplus_{\beta \in Q^{T}} M^{\left(\lambda^{0}+\beta\right)}$, where $\lambda^{0} \in \operatorname{supp} M$ is such that $d\left(\lambda^{0}\right)$ is maximal. The fact that $d\left(\lambda^{0}\right)$ is a maximum of $d$ implies that $\mathfrak{g}_{T}^{+} \cdot \Omega=0$. Thus $\Omega$ is a $\mathfrak{g}_{T}^{0} \oplus \mathfrak{g}_{T}^{+}$-module with trivial action of $\mathfrak{g}_{T}^{+}$. The inclusion $\Omega \subset M$ extends to a morphism of $\mathfrak{g}$-modules $\phi: M_{T}(\Omega) \rightarrow M$. As $M$ is simple, $\phi$ is onto and $\phi\left(L_{T}(\Omega)\right)=0$. Hence we have $M \simeq L_{T}(\Omega)$. By Corollary 2.4,(iii), $\Omega$ is simple. Finally, the support of $\Omega$ is invariant by $G\left(C_{M}^{1}\right)$ which is a subgroup of finite index in $Q^{I}$, hence $\Omega$ is cuspidal. The uniqueness of $\Omega$ follows from Corollary 2.4,(ii).

Corollary 3.7. For a simple generalized weight $\mathfrak{g}$-module $M$, the following assertions are equivalent:

(i) $M$ is cuspidal;

(ii) $C_{M}=Q$;

(iii) $M$ is dense.

\section{A cohomological Characterization of Finite Cuspidal modules}

The main result of this section, Proposition 4.3, is analogous to cohomological characterizations of cuspidal representations of $p$-adic groups (vanishing of the Jacquet functors) and of finite Chevalley groups (vanishing of Harish-Chandra restrictions). It enhances Theorem 3.6. Proposition 4.3 will not be used in the rest of the paper.

Throughout section $4, M$ is assumed to be finite. An admissible $\mathfrak{g}$-module (or $\mathfrak{g}_{0}$-module) is by definition a finite generalized weight $\mathfrak{g}$-module (or $\mathfrak{g}_{0}$-module) such that the dimensions of its generalized weight spaces are uniformly bounded.

Lemma 4.1. Let $M$ be cuspidal.

(i) Let $\alpha \in \operatorname{inj} M$ and let $x \in \mathfrak{g}_{0}^{(\alpha)}$ act injectively on $M$. Then there is $y \in$ $U\left(\mathfrak{g}_{0}\right)^{(-\alpha)}$ such that $x \cdot y$ acts bijectively on $M$.

(ii) $M$ is admissible.

Proof. By Theorem 3.6,(ii), $C_{M}^{1}$ is a group. Hence for some $\beta_{1}, \ldots, \beta_{k} \in C_{M}^{1}, \alpha+$ $\beta_{1}+\ldots+\beta_{k}=0$. Choose elements $x_{i} \in \mathfrak{g}_{0}^{\left(\beta_{i}\right)}$ which act injectively on $M$, and set $y=x_{1} \cdot \ldots \cdot x_{k}$. Then $x \cdot y$ acts injectively on $M$ and maps each generalized weight space to itself. Hence $x \cdot y$ acts bijectively on $M$. Therefore $x$ itself acts bijectively and the function $\lambda \mapsto \operatorname{dim} M^{(\lambda)}$ is $C_{M}^{1}$ invariant. By Proposition 3.2, the support of $M$ is a finite union of $C_{M}^{1}$-cosets, hence $M$ is admissible. 
Lemma 4.2. Let $\mathfrak{u}$ be a nilpotent Lie superalgebra and let $N$ be a $\mathfrak{u}$-module. Assume that $\mathfrak{u}_{0}$ contains an element $x$ which acts bijectively on $N$. Then

$$
H^{*}(\mathfrak{u}, N)=H_{*}(\mathfrak{u}, N)=0 .
$$

Proof. Set $\mathfrak{v}=\mathfrak{u} /(K x)$ and let $\Lambda^{*} \mathfrak{v}$ be the exterior superalgebra of $\mathfrak{v}$. There is a spectral sequence, [HS], computing $H_{*}(\mathfrak{u}, N)$ and such that

$$
E_{p, q}^{1}=H_{p}\left(K x, C^{q} \mathfrak{v} \otimes N\right) .
$$

As $x$ acts bijectively on $C^{q} \mathfrak{v} \otimes M$, we have $E_{p, q}^{1}=0$. Hence $H_{*}(\mathfrak{u}, N)=0$. The argument for the vanishing of $H^{*}(\mathfrak{g}, N)$ is similar.

Proposition 4.3. The following are equivalent:

(i) $M$ is cuspidal;

(ii) $H_{0}\left(\mathfrak{g}_{T}^{+}, M\right)=0$ for every proper triangular decomposition $T$;

(iii) $H^{0}\left(\mathfrak{g}_{T}^{+}, M\right)=0$ for every proper triangular decomposition $T$;

(iv) $H_{*}\left(\mathfrak{g}_{T}^{+}, M\right)=H^{*}\left(\mathfrak{g}_{T}^{+}, M\right)=0$ for every proper triangular decomposition $T$.

Proof. Let $T$ be a triangular decomposition. By Corollary 2.4, we have

$$
H^{0}\left(\mathfrak{g}_{T}^{+}, L_{T}(\Omega)\right)=\Omega
$$

for any simple object $\Omega$ of $\mathcal{G} W\left(\mathfrak{g}_{T}^{0}\right)$. Hence Assertion (iii) implies Assertion (i). Similarly, Assertion (ii) implies Assertion (i). Obviously Assertion (iv) implies Assertion (ii) and (iii).

Assume that Assertion (i) holds, and let $l: Q \rightarrow \mathbf{Z}$ be a linear function defining the triangular decomposition $T$. As the monoid generated by $\operatorname{inj} M$ is a finite index subgroup of $Q$, we have $l(\alpha)>0$ for some $\alpha \in \operatorname{inj} M$. By Lemma $4.1, \mathfrak{g}_{T}^{+}$contains a generalized root vector acting bijectively on $M$. Therefore Lemma 4.2 implies Assertion (iv).

\section{The small Weyl group}

To be able to further strengthen the claim of Theorem 3.6 we will assume throughout sections 5 and 6 that $\mathfrak{g}_{0}$ is reductive. Let $M$ be simple. By Proposition 2.2, (ii), $M$ is automatically a weight module. By definition, the small Weyl group of $M$ is the subgroup $W_{M}$ of the Weyl group $W$ of $\mathfrak{g}_{0}$ generated by the reflections $s_{\alpha}$, where $\alpha$ runs over $\Delta_{M}^{F} \cap \Delta_{0}$. By $Q_{M}^{F}$ and $Q_{M}^{I}$ we denote respectively the saturations of the subgroups of $Q$ generated by $\Delta_{M}^{F} \cap \Delta_{0}$ and $\Delta_{M}^{I}$.

Lemma 5.1. (i) The sets $\operatorname{supp} M$ and $C_{M}$ are $W_{M}$-invariant.

(ii) Any $\beta \in Q_{M}^{I}$ is $W_{M}$-invariant.

(iii) $Q_{M}^{F} \cap C_{M}=0$.

Proof. For $\alpha \in \Delta_{M}^{F}$, there is a subalgebra $\mathfrak{s}$ in $\mathfrak{g}_{0}$ isomorphic to $\mathfrak{s l}(2)$ with roots $\pm \alpha$. Since $M$ is locally finite as an $\mathfrak{s}$-module, Assertion (i) follows.

To prove Assertion (ii), fix $\alpha \in \Delta_{M}^{F}$ and $\beta \in Q_{M}^{I}$. As $\beta-s_{\alpha}(\beta)$ is a multiple of $\alpha$ and belongs to $Q_{M}^{I}$, we have $\beta-s_{\alpha}(\beta)=0$. Hence $\beta$ is $W_{M}$-invariant.

For $\alpha \in \Delta_{M}^{F}$, we have $\alpha+s_{\alpha}(\alpha)=0$. Therefore $\sum_{w \in W_{M}} w(\beta)=0$ for any $\beta \in Q_{M}^{F}$. Hence $Q_{M}^{F} \cap C_{M}$ is a subgroup of $C_{M}$, and $Q_{M}^{F} \cap C_{M} \subset Q_{M}^{I}$. But since $Q_{M}^{F}$ contains no $W_{M}$-invariant vectors, we have $Q_{M}^{F} \cap Q_{M}^{I}=0$ and therefore also $Q_{M}^{F} \cap C_{M}=0$. 
Set $\left(\mathfrak{g}_{0}\right)_{M}^{F}:=\mathfrak{h} \oplus\left(\bigoplus_{\alpha \in Q_{M}^{F}} \mathfrak{g}_{0}^{\alpha}\right),\left(\mathfrak{g}_{0}\right)_{M}^{+}:=\mathfrak{g}_{M}^{+} \cap \mathfrak{g}_{0},\left(\mathfrak{g}_{0}\right)_{M}^{I}:=\mathfrak{g}_{M}^{I} \cap \mathfrak{g}_{0}$ and $\mathfrak{p}(M):=\left(\left(\mathfrak{g}_{0}\right)_{M}^{F}+\left(\mathfrak{g}_{0}\right)_{M}^{I}\right) \oplus \mathfrak{g}_{M}^{+}$. For any Lie algebra $\mathfrak{a}$, put $\mathfrak{a}^{\prime}:=[\mathfrak{a}, \mathfrak{a}]$.

Corollary 5.2. (i) $\left(\mathfrak{g}_{0}\right)_{M}^{F}$ is a reductive Lie subalgebra of $\mathfrak{g}$, its root system is $\Delta_{M}^{F} \cap \Delta_{0}$ and its Weyl group is $W_{M}$.

(ii) The Lie algebras $\left(\left(\mathfrak{g}_{0}\right)_{M}^{F}\right)^{\prime}$ and $\left(\left(\mathfrak{g}_{0}\right)_{M}^{I}\right)^{\prime}$ commute.

(iii) $\mathfrak{p}(M)$ is a parabolic subalgebra of $\mathfrak{g}_{0}$.

Proof. Assertion (i) is a direct consequence of the definition of $\left(\mathfrak{g}_{0}\right)_{M}^{F}$, and Assertions (ii) and (iii) follow from the fact that $Q_{M}^{F} \cap C_{M}=0$, as proved in Lemma 5.1 .

For any triangular decomposition $T$, set $\mathfrak{p}(T):=\left(\mathfrak{g}_{T}^{0} \oplus \mathfrak{g}_{T}^{+}\right) \cap \mathfrak{g}_{0}$ and note that $\mathfrak{p}(T)$ is a parabolic subalgebra of $\mathfrak{g}_{0}$.

Proposition 5.3. Let $T, T^{\prime}$ be triangular decompositions, and let $\Omega$ and $\Omega^{\prime}$ be respectively a cuspidal $\mathfrak{g}_{0}^{T}$-module and a cuspidal $\mathfrak{g}_{0}^{T^{\prime}}$-module so that $M=L_{T}(\Omega) \simeq$ $L_{T^{\prime}}\left(\Omega^{\prime}\right)$. Then the parabolic subalgebras $\mathfrak{p}(T)$ and $\mathfrak{p}\left(T^{\prime}\right)$ are conjugated under $W_{M}$.

Proof. We have $\left(\mathfrak{g}_{0}\right)_{M}^{I} \oplus\left(\mathfrak{g}_{0}\right)_{M}^{+} \subset \mathfrak{p}(T) \subset \mathfrak{p}(M)$. It follows from Corollary 4.2 that the parabolic subalgebra $\mathfrak{p}(T)$ is determined by its intersection with $\left(\mathfrak{g}_{0}\right)_{M}^{I}$ which is a Borel subalgebra of $\left(\mathfrak{g}_{0}\right)_{M}^{I}$. As any two such intersections are conjugated by $W_{M}$, the Proposition is proved.

\section{Reduction of THE CLASSIFICATION PROBLEM}

Denote by $\mathcal{G}$ the set of all good triangular decompositions $T$ of $\mathfrak{g}$ (with $\mathfrak{g}_{T}^{0}$ containing the fixed Cartan subsuperalgebra $\mathfrak{h}$ ), and let $\mathcal{P}$ be the set of all parabolic subalgebras in $\mathfrak{g}_{0}$ which contain $\mathfrak{h}_{0}$. We define a canonical set of good triangular decompositions as a $W$-invariant subset $\mathcal{C} \subset \mathcal{G}$ such that the map $T \mapsto \mathfrak{p}(T)$ is a bijection between $\mathcal{C}$ and $\mathcal{P}$. For any classical simple Lie superalgebra one can define explicitly at least one canonical set of good triangular decompositions. This is an easy consequence of the classification of all good Levi subsuperalgebras given in the next section. Here are two examples. For instance, if $\mathfrak{g}=\mathfrak{p s q}(n)$, (see section 7 ; in $[\mathrm{K}] \mathfrak{p s q}(n)$ is denoted by $Q(n-1))$, then $\Delta_{0}=\Delta_{1}$ and therefore $\mathcal{G}$ itself is a canonical set of good triangular decompositions. If $\mathfrak{g}=\mathfrak{s l}(m \mid n)$ (see [K]), there is a natural Z-grading of $\mathfrak{g}$ as $\mathfrak{g}^{-1} \oplus \mathfrak{g}^{0} \oplus \mathfrak{g}^{1}\left(\right.$ where $\mathfrak{g}^{0}=\mathfrak{g}_{0}$ ), and for $m \neq n$ the set of all triangular decompositions $T$ such that $\mathfrak{g}_{T}^{+} \supset \mathfrak{g}_{1}$ is a canonical set of good triangular decompositions.

Assume now that $\mathcal{C}$ is any fixed canonical set of triangular decompositions. Here is a stronger version of Theorem 3.6.

Theorem 6.1. Let $M$ be simple.

(i) There is a triangular decomposition $T \in \mathcal{C}$ and a cuspidal $\mathfrak{g}_{T}^{0}$-module $\Omega$ such that $M \simeq L_{T}(\Omega)$.

(ii) For any $T^{\prime} \in \mathcal{C}$ and any cuspidal $\mathfrak{g}_{T^{\prime}}^{0}$-module $\Omega^{\prime}$ such that $M \simeq L_{T^{\prime}}\left(\Omega^{\prime}\right)$, there is $w \in W_{M}$ for which $T^{\prime}=T^{w}, \Omega^{\prime}=\Omega^{w}$.

Proof. Theorem 6.1 follows from Theorem 3.6 and Proposition 5.3.

Theorem 6.1 reduces the general classification problem to the problem of classifying cuspidal representations. The results of [M] together with Proposition 6.3 below provide a classification of finite cuspidal representations of $\mathfrak{g}$ up to a finite indeterminacy. 
The following lemma is proved in [M] (Lemma 3.3):

Lemma 6.2. Any admissible $\mathfrak{g}_{0}$-module has finite length.

Setting $\Omega^{e}:=\operatorname{ind}\left(\mathfrak{g}_{0}, \mathfrak{g} ; \Omega\right)$ for any $\mathfrak{g}_{0}$-module $\Omega$, we have now

Proposition 6.3. (i) For any finite cuspidal $\mathfrak{g}_{0}$-module $\Omega, \Omega^{e}$ contains at least one and only finitely many non-isomorphic cuspidal submodules.

(ii) For any finite cuspidal $\mathfrak{g}$-module $M$, there is at least one and only finitely many non-isomorphic cuspidal $\mathfrak{g}_{0}$-modules $\Omega$ such that $M \subset \Omega^{e}$.

Proof. Note first that if $\Omega$ is a finite cuspidal $\mathfrak{g}_{0}$-module, for any $\alpha \in \Delta_{0}$ there is an element $e_{\alpha} \in \mathfrak{g}_{0}^{\alpha}$ which acts bijectively on both $\Omega$ and $\Omega^{e}$. Furthermore $\Omega^{e}$ is an admissible $\mathfrak{g}_{0}$-module and is therefore of finite length by Lemma 6.2. Thus $\Omega^{e}$ may only have finitely many non-isomorphic irreducible $\mathfrak{g}$-submodules. Each of them is necessarily cuspidal since by Lemma $4.1 e_{\alpha}$ acts bijectively on it. Assertion (i) is proved. The proof of Assertion (ii) is similar.

\section{A Classification of CUSPidal Levi Subsuperalgebras}

In this section, we will use Kac's classification, $[\underline{K}$, to classify all cuspidal Levi subsuperalgebras (see the Introduction) of all simple classical Lie superalgebras (including the exceptional ones) and of $\mathbf{W}(n)$. For the remaining series $\mathbf{S}(n), \widetilde{\mathbf{S}}(n)$, $\mathbf{H}(n)$ the problem is still open.

The classification is based on the following three remarks:

(i) By Theorem 3.6, any cuspidal Levi subsuperalgebra is good. Furthermore, the map $\mathfrak{s} \mapsto \mathfrak{s}_{0}$ is a bijection between the set of good Levi subsuperalgebras of $\mathfrak{g}$ and the set of good Levi subalgebras of $\mathfrak{g}_{0}$. The inverse map is described as follows. Let $\mathfrak{s}_{0}$ be a good Levi subalgebra of $\mathfrak{g}_{0}$ and let $Q^{\prime}$ be the saturation in $Q$ of its root

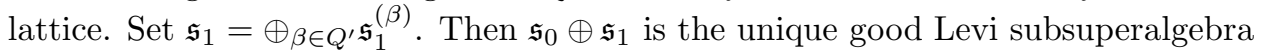
whose even part is $\mathfrak{s}_{0}$.

(ii) Let $\mathfrak{s}$ be a Levi subsuperalgebra such that $\mathfrak{s}_{0}$ is reductive. A result of Fernando (Theorem 5.2 in [Fe]) together with Proposition 6.3 implies that $\mathfrak{s}$ is cuspidal iff all simple factors of $\mathfrak{s}_{0}$ are of type $A$ or $C$.

(iii) If $\mathfrak{g}$ is a classical simple Lie superalgebra or $\mathfrak{g}=\mathbf{W}(n)$, and $\mathfrak{s}$ is a good Levi subsuperalgebra of $\mathfrak{g}$, then $\mathfrak{s}_{0}$ is reductive.

In the rest of this section, we present the list of all good Levi subsuperalgebras and the list of all cuspidal Levi subsuperalgebras. All simple Lie superalgebras which we do not define here are studied in detail in [K]. In addition, we will use the following notations:

- a partition $\mathbf{n}$ of a positive integer $n$ is a $k$-tuple of positive integers $\left(n_{1}, \ldots, n_{k}\right)$ with $\sum_{i} n_{i}=n$; the partition is proper iff $k \neq 1$;

- for a partition $\mathbf{n}$ we set

$\mathfrak{s l}(\mathbf{n}):=\left\{\left(g_{1}+\ldots+g_{k}\right) \in \mathfrak{g l}(\mathbf{n}):=\mathfrak{g l}\left(n_{1}\right) \oplus \ldots \oplus \mathfrak{g l}\left(n_{k}\right) \mid \sum_{i} \operatorname{tr}\left(g_{i}\right)=0\right\}$,

$\mathfrak{s q}(\mathbf{n}):=\left\{\left(g_{1}+\ldots+g_{k}\right) \in \mathfrak{q}(\mathbf{n}):=\mathfrak{q}\left(n_{1}\right) \oplus \ldots \oplus \mathfrak{q}\left(n_{k}\right) \mid \sum_{i} \operatorname{otr}\left(g_{i}\right)=0\right\}$,

$\mathfrak{p s q}(\mathbf{n}):=\mathfrak{s q}(\mathbf{n}) / K \cdot \mathrm{id}$,

where $\mathfrak{q}(l)$ is the Lie superalgebra of block matrices with $l \times l$-blocks

$$
\alpha=\left(\begin{array}{ll}
A & B \\
B & A
\end{array}\right)
$$

with arbitrary $l \times l$-matrices $A$ and $B$, and $\operatorname{otr} \alpha:=\operatorname{tr} B$. 
7.1. Let $\mathfrak{g}=\mathfrak{s l}(n \mid m)$, with $n>m$. Then $\mathfrak{g}_{0} \simeq \mathfrak{s l}(n, m)$ and $\mathfrak{g}_{1} \simeq U \otimes V^{*} \oplus U^{*} \otimes V$, where $U$ and $V$ are respectively the natural representations of $\mathfrak{g l}(n)$ and $\mathfrak{g l}(m)$. Any good Levi subsuperalgebra is a Lie subalgebra of $\mathfrak{g}_{0}$, it is necessarily cuspidal, and moreover it is isomorphic to $\mathfrak{s l}(\mathbf{n} \cup \mathbf{m})$ where $\mathbf{n}, \mathbf{m}$ are partitions of $n$ and $m$ respectively.

7.2. Let $\mathfrak{g}=\mathfrak{p s l}(n \mid n)$. Then $\mathfrak{g}_{0} \simeq \mathfrak{s l}(n) \oplus \mathfrak{s l}(n)$ and $\mathfrak{g}_{1} \simeq U \otimes V^{*} \oplus U^{*} \otimes V$, where $U$ and $V$ are respectively the natural representations of the two factors $\mathfrak{s l}(n)$. Any good Levi subsuperalgebra is cuspidal, and moreover it is isomorphic either to $\mathfrak{g}$ or to $\mathfrak{s l}(\mathbf{n}) \oplus \mathfrak{s l}\left(\mathbf{n}^{\prime}\right), \mathbf{n}$ and $\mathbf{n}^{\prime}$ being partitions respectively of $n$ such that at least one of them is proper.

7.3. Let $\mathfrak{g}=\mathfrak{o s p}(n \mid 2 m)$. Then $\mathfrak{g}_{0} \simeq \mathfrak{o}(n) \oplus \mathfrak{s p}(2 m)$ and $\mathfrak{g}_{1} \simeq U \otimes V$, where $U$ and $V$ are respectively the natural representations of $\mathfrak{o}(n)$ and $\mathfrak{s p}(2 m)$. Any good Levi subsuperalgebra is of the type $\mathfrak{o s p}\left(n^{\prime} \mid 2 m^{\prime}\right) \oplus \mathfrak{s l}(\mathbf{n}) \oplus \mathfrak{s l}(\mathbf{m})$, where $n-n^{\prime}$ is even, $\mathbf{n}$ and $\mathbf{m}$ are respectively partitions of $n-n^{\prime}$ and $m-m^{\prime}$. Such a subsuperalgebra is cuspidal iff $n^{\prime} \leq 6$. The latter follows from the fact that $\mathfrak{o}(k)$ is of type $A$ or $C$ for $k \leq 6$.

7.4. Let $\mathfrak{g}=\mathbf{s p}(n)$, see $\left[\mathrm{P}\right.$ (in $[\mathrm{K}] \mathbf{s p}(n)$ is denoted by $P(n-1)$ ). Then $\mathfrak{g}_{0} \simeq$ $\mathfrak{s l}(n)$ and $\mathfrak{g}_{1} \simeq S^{2} V \oplus \wedge^{2} V^{*}$, where $V$ is the natural representation of $\mathfrak{s l}(n)$. Any good Levi subsuperalgebra is cuspidal. If $n$ is odd, all such subsuperalgebras are isomorphic to $\mathfrak{g}$ or to $\mathfrak{s l}(\mathbf{n})$, where $\mathbf{n}$ is a proper partition of $n$. If $n$ is even, all such subsuperalgebras are isomorphic to $\mathfrak{g}$, to $\mathfrak{s l}(\mathbf{n})$ for a proper partition $\mathbf{n}$ of $n$ other than $(n / 2, n / 2)$, or to $\mathfrak{s l}(n / 2 \mid n / 2)$.

7.5. Let $\mathfrak{g}=\mathfrak{p s q}(n)$. Then $\mathfrak{g}_{0} \simeq \mathfrak{s l}(n)$ and $\mathfrak{g}_{1}$ is isomorphic to the adjoint representation. Any good Levi subsuperalgebra cuspidal and is isomorphic to $\mathfrak{p s q}(\mathbf{n})$, where $\mathbf{n}$ is a partition of $n$.

7.6. Let $\mathfrak{g}=\mathbf{D}(2,1, \alpha)$. Then $\mathfrak{g}_{0} \simeq \mathfrak{s l}(2) \oplus \mathfrak{s l}(2) \oplus \mathfrak{s l}(2)$ and $\mathfrak{g}_{1} \simeq V^{1} \otimes V^{2} \otimes$ $V^{3}$, where $V^{1}, V^{2}$ and $V^{3}$ are respectively the natural representations of the three factors. Any good Levi subsuperalgebra is cuspidal and is isomorphic to $\mathfrak{g}$ or to any proper Levi subalgebra of $\mathfrak{g}_{0}$.

7.7. Let $\mathfrak{g}=\mathbf{G}(3)$. Then $\mathfrak{g}_{0} \simeq \mathfrak{s l}(2) \oplus \mathbf{G}_{2}$ and $\mathfrak{g}_{1} \simeq V \otimes U, V$ being the natural representation of $\mathfrak{s l}(2)$ and $U$ being the 7 -dimensional $\mathbf{G}_{2}$-module. Any good Levi subsuperalgebra is isomorphic to one of the following:

(i) $\mathfrak{g}$;

(ii) $\mathfrak{g l}(1) \oplus \mathbf{G}_{2}$;

(iii) a Levi subalgebra of $\mathfrak{g}_{0}$ which does not contain a short root of $\mathbf{G}_{2}$;

(iv) a Levi subalgebra of $\mathfrak{g}_{0}$ which is isomorphic to $\mathfrak{s l}(2,1,1)$ and contains a long root of $\mathbf{G}_{2}$;

(v) $\mathfrak{o}(4) \ltimes H_{4}$, where $H_{4}$ is the Heisenberg superalgebra of dimension $1 \mid 4$.

The good cuspidal Levi subsuperalgebras are the ones of types (iii), (iv) and (v).

7.8. Let $\mathfrak{g}=\mathbf{F}(4)$. Then $\mathfrak{g}_{0} \simeq \mathfrak{s l}(2) \oplus \mathfrak{o}(7)$ and $\mathfrak{g}_{1} \simeq V \otimes U, V$ being the natural representation of $\mathfrak{s l}(2)$ and $U$ being the 8-dimensional spin representation of $\mathfrak{o}(7)$. Any good Levi subsuperalgebra is isomorphic to one of the following:

(i) $\mathfrak{g}$;

(ii) $\mathfrak{g l}(1) \oplus \mathfrak{o}(7)$;

(iii) $\mathfrak{o s p}(4 \mid 2)$; 
(iv) a Levi subalgebra of $\mathfrak{g}_{0}$ which does not contain $\mathfrak{s l}(2) \oplus \mathfrak{s l}(2) \oplus \mathfrak{s l}(2)$.

The good cuspidal Levi subsuperalgebras are the ones of types (iii) and (iv).

7.9. Let $\mathfrak{g}=\mathbf{W}(n)$. Then $\mathfrak{g l}(n)$ is embedded canonically into $\mathfrak{g}_{0}$. Any good Levi subsuperalgebra is simply a Levi subalgebra of $\mathfrak{g l}(n)$ and is necessarily cuspidal. Moreover, such a subalgebra is isomorphic to $\mathfrak{g l}(\mathbf{n})$ for an arbitrary partition $\mathbf{n}$ of $n$.

\section{Conclusion}

The present paper, together with $[\mathrm{M}]$ provides the following classification results for simple finite weight $\mathfrak{g}$-modules.

8.1. A complete classification of simple finite weight modules over any reductive Lie algebra $\mathfrak{g}$. A simple finite module of $\mathfrak{g}$ is isomorphic to a tensor product of simple finite weight modules over the simple components of $\mathfrak{g}$, and for a simple Lie algebra the classification is carried out explicitly in [M].

Moreover, if $\mathfrak{g}$ is any finite dimensional Lie superalgebra with reductive even part, Proposition 6.3 reduces the classification of simple finite weight modules to a finite indeterminacy.

8.2. A complete classification of simple finite weight modules over $\mathfrak{s l}(m \mid n)$ with $m \neq n$ and over $\mathbf{W}(n)$. Indeed, if $\mathfrak{g}=\mathfrak{s l}(m \mid n)$ or $\mathfrak{g}=\mathbf{W}(n)$, there is a natural Z-grading of $\mathfrak{g}$ as $\mathfrak{g}^{-1} \oplus \mathfrak{g}^{0} \oplus \mathfrak{g}^{1} \oplus \ldots$, where $\mathfrak{g}^{0} \simeq \mathfrak{s l}(m, n)$ for $\mathfrak{g}=\mathfrak{s l}(m \mid n)$, and $\mathfrak{g}^{0} \simeq \mathfrak{g l}(n)$ for $\mathfrak{g}=\mathbf{W}(n)$. Moreover, when $m \neq n$, the map $M \mapsto H^{0}\left(\mathfrak{g}^{-1}, M\right)$ is a bijection between all simple weight $\mathfrak{g}$-modules (modulo the parity change) and all simple weight $\mathfrak{g}_{0}$-modules. Thus all simple finite weight $\mathfrak{g}$-modules are classified.

8.3. A complete classification of non-cuspidal simple finite weight modules over $\mathfrak{p s l}(n \mid n), \mathbf{s p}(2 k+1)$ and $\mathbf{D}(2,1, \alpha)$. It follows from the results in sections 6 and 7. More generally, if $\mathfrak{g}$ is any Lie superalgebra whose even part $\mathfrak{g}_{0}$ is reductive, we obtain a complete classification of all finite simple weight modules $M$ with $\mathfrak{g}_{M}^{I} \subset \mathfrak{g}_{0}$.

In order to finish the classification of finite simple weight modules over all simple classical Lie superalgebras and over $\mathbf{W}(n)$, it is necessary to classify the finite cuspidal modules over $\mathfrak{p s l}(n \mid n), \mathfrak{o s p}(n \mid 2 m)$ (with $n \leq 6), \mathfrak{p s q}(n), \mathbf{D}(2,1, \alpha), \mathbf{G}(3)$, and $\mathfrak{o}(4) \ltimes H_{4}$. For the remaining three Cartan-type series of simple Lie superalgebras $\mathbf{S}(n), \widetilde{\mathbf{S}}(n), \mathbf{H}(n)$ one should first classify their cuspidal Levi subsuperalgebras and then try to complete the classification.

\section{REFERENCES}

[CFO] A. Cylke, V. Futorny and S. Ovsienko, On the support of irreducible non-dense modules for finite-dimensional Lie algebras, Canad. J. Math. (to appear).

[Fe] S. Fernando, Lie algebra modules with finite dimensional weight spaces I, TAMS 322 (1990), 757-781. MR 91c:17006

[Fu] V. Futorny, The weight representations of semisimple finite dimensional Lie algebras, Ph.D. Thesis, Kiev University, 1987.

[HS] G. Hochschild, J.-P. Serre, Cohomology of Lie algebras, Ann. Math. 57 (1953), 591-603. MR 14:943c

[K] V. Kac, Lie superalgebras, Adv. Math. 26 (1977), 8-96. MR 58:5803

$[\mathrm{M}] \quad$ O. Mathieu, Classification of irreducible weight modules, Ann. Inst. Fourier (to appear).

[P] I. Penkov, Characters of strongly generic irreducible Lie superalgebra representations, Internatl. J. Math. 9 (1998), 331-366. MR 99d:17011 
[PS] I. Penkov, V. Serganova, Generic irreducible representations of finite-dimensional Lie superalgebras, Internatl. J. Math. 5 (1994), 389-419. MR 95c:17015

[Sch] M. Scheunert, Invariant supersymmetric multilinear forms and the Casimir elements of P-type Lie superalgebras, J. Math. Phys. 28 (1987), 1180-1191. MR 89b:17008

Department of Mathematics, University of California at Riverside, Riverside, CalIFORNIA 92521

Current address: Department of Mathematics, University of California, Los Angeles, California 90095- 1555

E-mail address: dimitrov@math.ucla.edu

Université Louis Pasteur, IRMA, 7 rue René Descartes, 67000 Strasbourg, France

E-mail address: mathieu@math.u-strasbg.fr

Department of Mathematics, University of California at Riverside, Riverside, CalIFORNIA 92521

E-mail address: penkov@math.ucr.edu 